\section{Disappearing pancreatic arteriovenous malformation}

Arteriovenous malformation (AVM) of the pancreas is a rare disorder for which treatment is recommended as it may cause development of portal hypertension and increase the risk of gastrointestinal bleeding [1 -3].

A 50-year-old man was referred to our institute for examination of epigastric pain with mild elevation of serum amylase (143 U/L). There was no history of hemorrhagic diseases for the patient or his family. Using dynamic computed tomography (CT), an AVM was recognized at the pancreatic tail, and the return from the splenic vein was visible during the arterial phase ( Fig. 1 a,b). Color Doppler images from endoscopic ultrasonography (EUS) demonstrated numerous turbulent flows in the patchy hypoechoic parenchyma suggesting accompanying pancreatitis $(\bullet$ Fig. 2a). No variceal lesion was recognized during upper gastrointestinal endoscopy, nor was any extrapancreatic AVM identified by truncal CT or brain magnetic resonance imaging (MRI). Informed consent was obtained, and the patient chose careful follow-up.

Over the ensuing months, the patient's symptoms diminished and the serum amylase level normalized spontaneously. After 15 months, dynamic CT showed that the pancreatic AVM had disappeared $(\bullet$ Fig. $1 \mathbf{c}, \mathbf{d}$ ), and this was confirmed by EUS 6 months after that ( $\bullet$ Fig. 2 b).
Up till 2012, 81 cases of pancreatic AVM had been reported [3]. Symptoms included gastrointestinal bleeding (49\%) and abdominal and/or back pain (48\%), whereas $13 / 81$ (16\%) were asymptomatic cases. Treatments including surgical resection (62\%), transarterial embolization (17\%), and irradiation (2\%) were performed in the majority of cases. Meanwhile, 9/13 (69\%) asymptomatic cases were followed without treatment. To date, from a PubMed keyword survey, there has been no report of pancreatic AVM spontaneously disappearing during follow-up. Although further reliable evidence is needed, careful follow-up under fully informed consent may be feasible in cases of pancreatic AVM with a low risk of gastrointestinal bleeding.

\section{Endoscopy_UCTN_Code_CCL_1AZ_2AI}

\section{Competing interests: None}
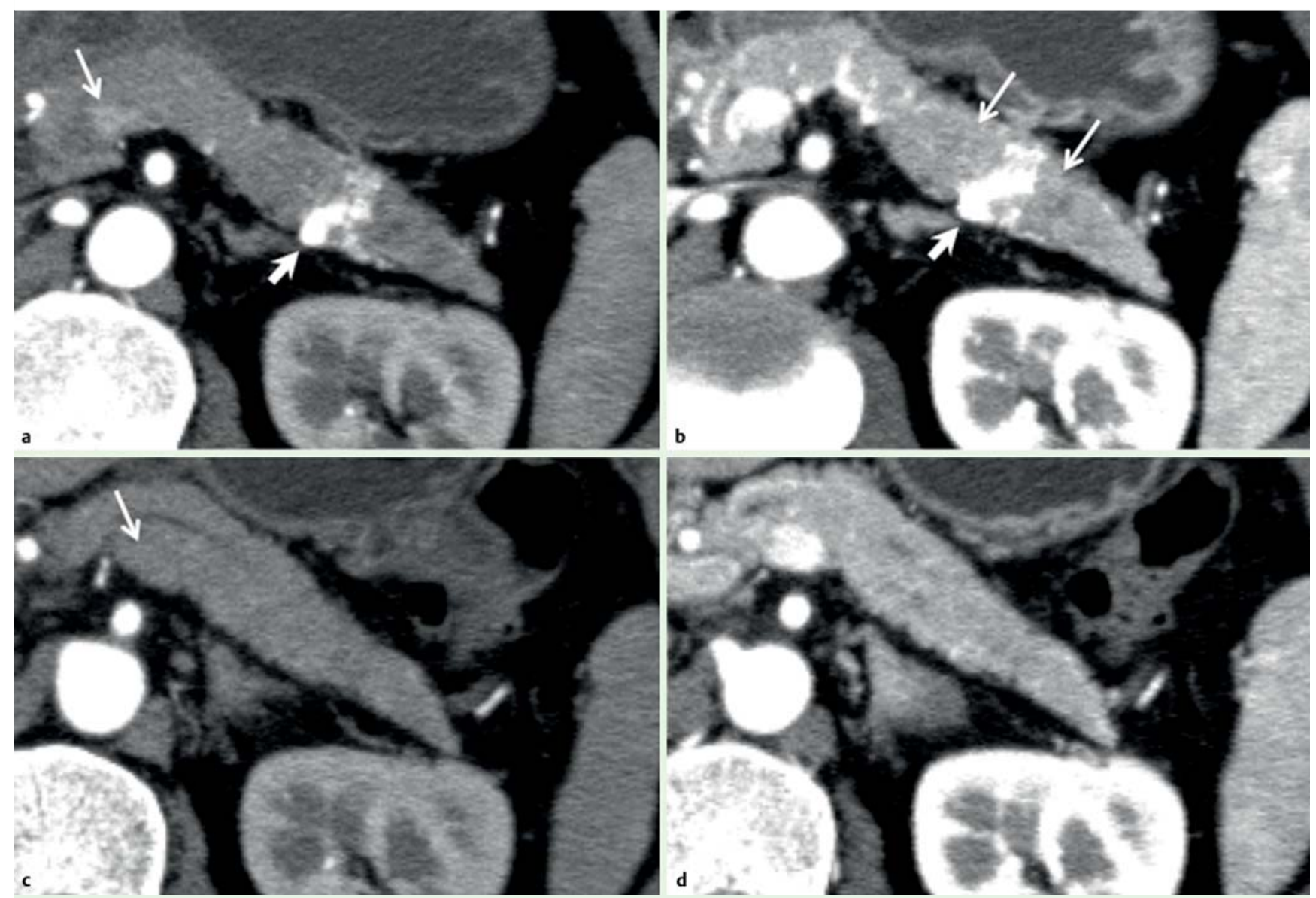

Fig. 1 Dynamic computed tomography (CT) of a 50-year-old man with an arteriovenous malformation (AVM): a an arterial phase image (29s) showing a highly vascular lesion at the pancreatic tail (thick arrow) and early venous return at the splenic vein (thin arrow); $\mathbf{b}$ a pancreatic parenchymal phase image (49s) showing increased flow volume in the AVM (thick arrow) and a low-density area with a poorly defined margin surrounding it (thin arrows). c Follow-up CT images at 15 months, demonstrating that the AVM had disappeared. The venous return during the arterial phase was now absent (c, thin arrow) but visible at the pancreatic parenchymal phase $(\mathbf{d})$. 

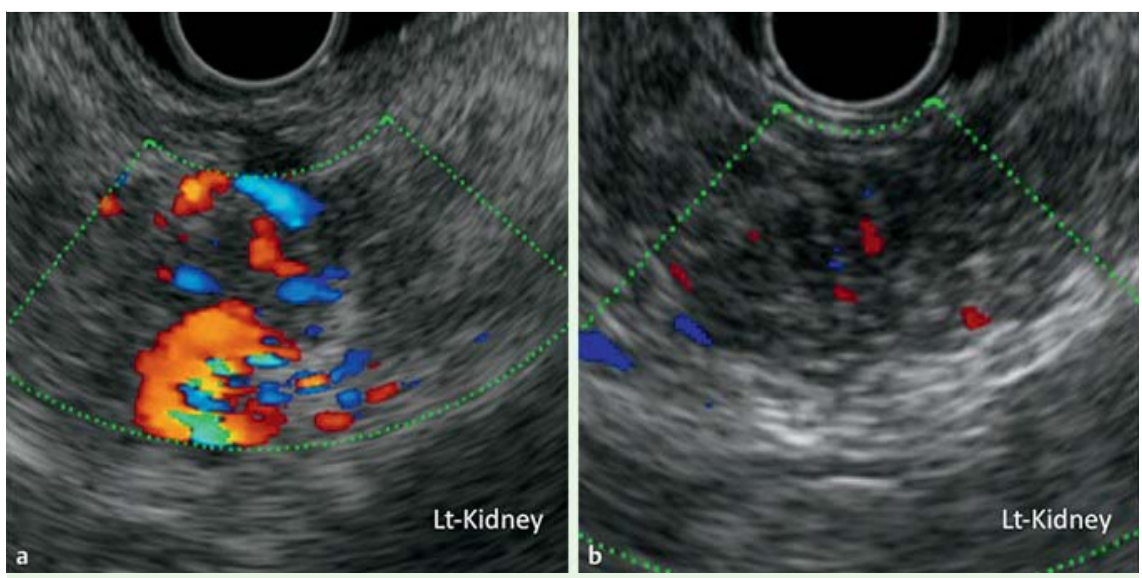

Fig. 2 Endoscopic ultrasonography (EUS) of a 50-year-old man with an arteriovenous malformation (AVM). a Pulsatile turbulent flows within the patchy hypoechoic pancreatic parenchyma confirmed in color Doppler images. b No pulsatile flows were present within the pancreatic lesion, 21 months later, when compared with the previous examination (a).
Bibliography

Dol http://dx.doi.org/ $10.1055 / \mathrm{s}-0034-1377594$ Endoscopy 2014; 46: E524-E525

(c) Georg Thieme Verlag KG Stuttgart · New York ISSN 0013-726X

Corresponding author Hiroyuki Matsubayashi, MD, PhD Division of Endoscopy Shizuoka Cancer Center 1007 Shimonagakubo Nagaizumi

Suntogun

Shizuoka

411-8777

Japan

Fax: +81-55-9895222

h.matsubayashi@scchr.jp
Hiroyuki Matsubayashi' ${ }^{1}$, Toru Matsui ${ }^{1}$, Kimihiro Igarashi ${ }^{1}$, Kinichi Hotta ${ }^{1}$, Kenichiro Imai ${ }^{1}$, Hideyuki Kanemoto², Hiroyuki Ono ${ }^{1}$

${ }^{1}$ Division of Endoscopy, Shizuoka Cancer Center, Shizuoka, Japan

${ }^{2}$ Division of Hepato-Biliary-Pancreatic Surgery, Shizuoka Cancer Center, Shizuoka, Japan

\section{References}

1 Koito K, Namieno T, Nagakawa $T$ et al. Congenital arteriovenous malformation of the pancreas: its diagnostic features on images. Pancreas 2001; 22: 267-273

2 Kanno A, Satoh K, Kimura Ket al. Acute pancreatitis due to pancreatic arteriovenous malformation: 2 case reports and review of the literature. Pancreas 2006; 32: 422 - 425

3 Song KB, Kim SC, Park JB et al. Surgical outcomes of pancreatic arteriovenous malformation in a single center and review of literature. Pancreas 2012; 41: 388-396 